\title{
Investigation on Therpeutic Efficacy of Teofilin in Calves with Respiratory Diseases
}

\author{
Bulent Elitok ${ }^{1 *}$ and Salih Sezer ${ }^{2}$ \\ ${ }^{1}$ Department of Internal Medicine, Afyon Kocatepe University, Turkey \\ ${ }^{2}$ Private Veterinarian, Turkey
}

Submission: July 10, 2018; Published: August 22, 2018

*Corresponding author: Bulent Elitok, Faculty of Veterinary Medicine, Department of Internal Medicine, Afyon Kocatepe University, 03200/ Afyonkarahisar, Turkey, Email: elitok1969@hotmail.com

\begin{abstract}
This study was carried out in a total of 50 calf calves in the Uşak province that were found to have developed a clinical respiratory system problem. Twenty-five animals constituted the control group (KG) while 25 animals constituted the experimental group (DG). KG calves were treated under the classical treatment procedure. In addition to the routine clinical treatment procedure for DG animals, intravenous (iv) theophylline (TECARß $240 \mathrm{mg} / 10 \mathrm{ml}$ iv ampul) is administered in the form of slow infusion in a physiological saline 2 times daily at a dose of $6 \mathrm{mg} / \mathrm{kg}$ for 3 days). Before and after starting work on all of the animals studied 1.2.3. Clinical, haematological and blood biochemical examinations were performed on days. With the application of theophylline, contraction of the muscles seen in some of the DG animals was formed, and no such symptom was observed in the control group animals. It has been observed that theophylline applications cause some significant changes in hematological parameters, especially in the number of RBCs. The most significant increases were obtained in terms of platelet (PLT). The mean levels of enzyme levels of aspartate aminotransferase (AST), alkaline phosphatase (ALP), sorbitol dehydrogenase (SDH), lactate dehydrogenase (LDH), glutamate dehydrogenase (GLDH), creatinine kinase (CK) decreased progressively in both groups on day 3 (p $<0.05)$, which was statistically significant in terms of all time periods in DG animals compared to KG. As a result; it has been determined that theophylline investigated for the first time in the refrigerator can provide significant improvements in the clinical symptoms of the respiratory system and contribute to recovery..
\end{abstract}

Keywords: Theophylline; Calf; Clinical; Hematologic; Blood biochemistry

Abbreviations: PLT: Platelet, AST: Aspartate Aminotransferase; ALP: Alkaline Phosphatase; SDH: Sorbitol Dehydrogenase; LDH: Lactate Dehydrogenase; GLDH: Glutamate Dehydrogenase; CK: Creatinine Kinase, COPD: Chronic Obstructive Pulmonary Disease

\section{Introduction}

Respiratory diseases in the calves cause economic losses in animal breeding [1-3]. This disease complex, which is of particular importance in feeder animals, especially on the ice, is formed by the combination of many factors [4-7]. Main specifications; cough, power breathing, stagnation, loss of appetite, nasal discharge, and abdominal solu- tion. Although the increase in body temperature may be at the beginning of the disease, it may subsequently fall to normal, but the pulse rate increases [5,8-10]

Theophylline is a methylxanthine group drug that is widely used as a bronchodilator in diseases such as asthma where respiratory tract constriction is severe [11-13]. The recommended therapeutic range for treatment efficacy is 10$20 \mu \mathrm{g} / \mathrm{ml}$ and reported to occur when side effects are generally used above $20 \mu \mathrm{g} / \mathrm{ml}$ [14].

The methylxanthine group of drugs, theophylline, is an effective bronchodilator for the treatment of airway obstruction diseases such as chronic obstructive pulmonary disease (COPD), asthma, neonatal apnea and bradycardia syndrome $[15,16]$. In addition to the bronchodilator effect of theophylline, diuretics and diuretic and respiratory muscle strengthening effects and antiinflammatory effects have been found to increase catecholamine release [17-19].

The aim of this study was to demonstrate the therapeutic efficacy of theophylline, a respiratory stimulant and bronchodilator, which was commonly used in the human medicine and less frequently used in the veterinary field, in respiratory system diseases.

\section{Materials and Methods}

\section{Animal materials}

The study was carried out in a total of 50 calves, 25 control groups (KG) and 25 experimental groups (DG), 0 to 6 months old, who were found to have developed a respiratory system problem clinically in Ușak city and according to the method 
[5]. KG calves were treated under the routine clinical treatment procedure (Clamoxy-LA®Pfizer, Injacom-C® Ceva, Nervit Kompoze® Vetaş, Fulimed®Alke).

In addition to the routine clinical treatment procedure for DG animals, intravenous (iv) theophylline (TECAR® $240 \mathrm{mg} / 10 \mathrm{ml}$ iv ampul-Avicenna (iv) is administered intravenously in the form of slow infusion in a physiological saline at a dose of $6 \mathrm{mg} / \mathrm{kg}$ for 2 days, Farma Foreign Trade Inc. and Istanbul-Turkey).

Respiratory quality (depth, superficiality), cough, nasal discharge, auscultation of pulmonary sounds were examined and recorded in addition to clinical examinations of $\mathrm{KG}$ and DG animals (body temperature, respiration and heart rates and rumen contractions at 5 minutes). Blood samples from V. jugularis were taken and examined for hematological and blood biochemical examinations on days of $1,2,3$.

This study was supported by the reference number 49015 of Afyon Kocatepe University Animal Experiments Ethics Committee with the reference number of AKUHADYEK and supported by the Coordination Unit of Scientific Research Projects of Afyon Kocatepe University (BAPK) with the reference number of 17.SAGBIL.15.

\section{Methods \\ Clinical Experiments}

The routine clinical examinations such as inspiration, body temperature, respiration and heart rates, number of ruminant contractions, strength before and after intravenous administration of theophylline, and the clinical laboratory examinations of the parameters described by Blood and Radostits (1989). The obtained data are recorded and maintained for statistical evaluation.

\section{Hematological Meausrements}

Blood was drawn into EDTA blood vessels on the 1st and 2nd day before and after starting the use of theophylline by i.v in the subsequent control group and study group animals, and blood samples were sent to the laboratory on the same day and as soon as possible. These blood samples were counted in the blood count using counting solutions. The obtained data were recorded and evaluated at later stages for comparison with the control group values. In blood samples taken for hematological examination; (RBC), total leukocyte (WBC), hematocrit (HCT), hemoglobin (HB), mean corpuscular volume (MCV), mean corpuscular hemoglobin (MHC), mean corpuscular hemoglobin concentration (MCHC), lymphocyte (LENF), neutrophil Hematological examinations such as NOTR, eosinophil (EOS), monocyte (MON) and basophil (BAZ) were measured using commercial test kits with a Chemray Brand blood count device.

\section{Serum biochemical measurements}

For this purpose, blood samples were taken from the V. jugularis before and after the administration of i.v theophylline and on the 2 nd and 3rd days in the animals designated as control and study groups. Blood samples were taken from the laboratory, where the serum was removed and the extracted sera were stored at +4 degrees centigrade, taking gods if not measured immediately. In blood biochemical examinations; Serum glutamate dehydrogenase (SDH) (spectrophotometrically measured at a wavelength of $340 \mathrm{~nm}$ in a Chemwell Mark apparatus), serum glutamate dehydrogenase (GLDH) (Chemwell Elisa (R)), serum aspartate aminotransferase (AST), serum gamma glutamyltransferase (GGT), serum lactate dehydrogenase (TP), Albumin (ALB), Glucose (GLU), Total and Direct Bilirubin (TB and $\mathrm{DB}$ ) were measured using commercial kit in the Chemwell Brand autoanalyser with the levels of creatinine kinase (CK).

\section{Statistical analyses}

The measurements referred to in all of the animals that make up the study material were repeated on the 1st and 2nd days after the control and study. Statistical calculations of the groups were made according to the ANOVA method. The Duncan test was used to determine the intraparticular differences in the study group. Statistical analyzes were performed using the Windows-compatible SPSS 18.0 (Inc. Chicago, II, USA) package program. Data were presented as mean \pm standard error and $p$ $<0.05$ was considered significant.

\section{Results}

A total of 50 calves (control $n=25$, study group $n=25$ ) aged between 68 and 82 days were used in this study. The mean age of the patients was $72 \pm 2$ days in DG and $70 \pm 3$ days in DG. There was no statistical difference between group mean age ( $p>$ 0.05 ). Of the 25 animals that make DG, 10 are female, 15 are male calves, and KG is 8 female and 17 male calves. Findings from repeated measurements on day 1 and day 2 before and after the start of the study, as shown below; clinical, hematological and biochemical findings.

\section{Clinical findings}

Body temperature decreased significantly $(\mathrm{p}<0.05)$ from the statistical point of view in the later time periods but there was no difference statistically between the two groups in terms of body temperature ( $p>0.05$ ). However, when compared to KG; respiratory and cardiac frequencies were statistically significant $(\mathrm{p}<0.05)$ higher in DG animals.

When Table 1 is examined; There was no statistically significant difference between the body temperature (T), respiratory $(\mathrm{R})$ and heart $(\mathrm{P})$ frequencies in 5 minutes and 5 minute ruminal movements $(\mathrm{RH})$ in both groups ( $\mathrm{p}>0.05)$. However, in both groups it was concluded that the reference levels of VS were above the reference levels and that an infection situation was the case when other clinical findings were taken into consideration. When compared with UO; On the 1st day of US, VS averages decreased significantly $(p<0.05)$ in DG and KG animals, but there was no statistically significant difference ( $p>$ 0.05 ) between DG and KG averages in terms of this parameter. (P $<0.05$ ). However, there was no statistically significant difference 


\section{Juniper Online Journal of Case Studies}

( $p>0.05$ ) between the mean values at different time points of DG ( $p>0.05$ ). The differences between the groups in terms statistically significant ( $\mathrm{p}<.05)$, while the increase was not of time intervals and UO averages and US time intervals were statistically significant 0.05 ).

Table 1: Comparison of body temperature, heart and respiratory frequencies and ruminal movements of animals.

\begin{tabular}{|c|c|c|c|c|c|}
\hline Parameters & Group/Time & UÖ & US & US & US \\
\hline & & $\mathbf{( n = 2 5 )}$ & $\mathbf{1}^{\text {st }}$ Day & $\mathbf{2}^{\text {nd }}$ Day & $\mathbf{3}^{\text {rd }}$ Day \\
\hline \multirow{2}{*}{$\mathrm{T}\left({ }^{\circ} \mathrm{C}\right)$} & KG & $39.20 \pm 1.10^{\mathrm{a}}$ & $38.40 \pm 1.30^{\mathrm{ab}}$ & $38.10 \pm 1.14^{\mathrm{ab}}$ & $37.50 \pm 1.02^{\mathrm{b}}$ \\
\cline { 2 - 6 } & DG & $39.24 \pm 1.20^{\mathrm{a}}$ & $38.36 \pm 1.20^{\mathrm{ab}}$ & $38.00 \pm 1.10^{\mathrm{ab}}$ & $37.40 \pm 1.00^{\mathrm{b}}$ \\
\hline \multirow{2}{*}{$\mathrm{P}(\mathrm{freq} / \mathrm{min})$} & KG & $86.00 \pm 6.00^{\mathrm{b}}$ & $72.00 \pm 8.00^{\mathrm{c}}$ & $63.00 \pm 6.00^{\mathrm{d}}$ & $52.00 \pm 7.00^{\mathrm{e}}$ \\
\cline { 2 - 6 } & DG & $83.00 \pm 4.00^{\mathrm{b}}$ & $142 \pm 12.00^{\mathrm{a}}$ & $146 \pm 17.00^{\mathrm{a}}$ & $145 \pm 12.00^{\mathrm{a}}$ \\
\hline \multirow{2}{*}{$\mathrm{R}(\mathrm{freq} / \mathrm{min})$} & KG & $36.00 \pm 5.00^{\mathrm{d}}$ & $37.00 \pm 6.00^{\mathrm{d}}$ & $36.00 \pm 5.00^{\mathrm{d}}$ & $35.00 \pm 4.00^{\mathrm{d}}$ \\
\hline \multirow{2}{*}{$\mathrm{RH}$} & DG & $35.00 \pm 6.00^{\mathrm{d}}$ & $50.00 \pm 4.00^{\mathrm{c}}$ & $56.00 \pm 3.00^{\mathrm{b}}$ & $60.00 \pm 3.00^{\mathrm{a}}$ \\
\hline (move/5min) & KG & $5.00 \pm 3.00^{\mathrm{c}}$ & $6.00 \pm 3.00^{\mathrm{c}}$ & $8.00 \pm 2.00^{\mathrm{b}}$ & $10.00 \pm 2.00^{\mathrm{a}}$ \\
\hline
\end{tabular}

a-d . The difference between the averages of the control groups carrying different letters in the same column is important in terms of statistics $(p<0,05)$. KG: Control group, DG: Experiment group, UO: Before experiment, US: After experiment.

\section{Hematological findings}

The averages and comparisons of the haematological parameters of UO and US groups are given in Table 2. When this table is examined; it has been observed that theophylline applications lead to significant changes in hematological parameters, especially in the number of RBCs. According to the results obtained from the measurements made; Similarly, the decrease in the mean of RBC, HB, HTC and WBC levels in the US period was statistically significant in terms of time in the US period ( $\mathrm{p}<0.05$ ) ( $\mathrm{p} \& \mathrm{lt}$; 0.05). A similar situation was also found in terms of NOTR and EOS averages and the difference was statistically significant ( $p<0.05)$. On the contrary, when the average of the LENF and MON levels in the US period was significantly higher $(\mathrm{p}<0.05)$ in terms of statistical significance than that of the UH turnover, when this height was compared with KG, the highest levels were obtained in terms of statistics and gradually increased with time $(\mathrm{p}<0.05)$.

The most significant increases were obtained in terms of platelet (PLT). When Table 2 is examined; $(\mathrm{P}<0.05)$, the highest level was obtained on day 3 , the last day of the study, and the difference between the time intervals and the groups was statistically significant ( $\mathrm{p}<0.05$ ), while the PLT average did not differ between the groups 0.05 ).

Table 2: Hematological findings.

\begin{tabular}{|c|c|c|c|c|c|c|c|c|c|c|c|c|c|c|}
\hline \multirow[t]{2}{*}{ Time } & Groups & $\begin{array}{c}\text { WBC } \\
\left(10^{3} /\right. \\
\mu \mathrm{L})\end{array}$ & $\begin{array}{c}\text { RBC } \\
\left(10^{6} /\right. \\
\mu \mathrm{L})\end{array}$ & $\begin{array}{c}\text { HGB } \\
(\mathrm{g} / \mathrm{dL})\end{array}$ & $\begin{array}{l}\text { HCT } \\
(\%)\end{array}$ & $\begin{array}{l}\text { MCV } \\
\text { (fl) }\end{array}$ & $\begin{array}{l}\text { MCH } \\
\text { (g/dL) }\end{array}$ & $\begin{array}{c}\text { PLT } \\
\left(10^{9} / \mathrm{L}\right)\end{array}$ & $\begin{array}{c}\text { MCHC } \\
\text { (pg) }\end{array}$ & $\begin{array}{c}\text { LENF } \\
\left(10^{3} /\right. \\
\mu \mathrm{L})\end{array}$ & $\begin{array}{c}\text { MONO } \\
\left(10^{3} /\right. \\
\mu \mathrm{L})\end{array}$ & $\begin{array}{c}\text { NOTR } \\
\left(10^{3} /\right. \\
\mu \mathrm{L})\end{array}$ & $\begin{array}{c}\text { EOS } \\
\left(10^{3} /\right. \\
\mu L)\end{array}$ & $\begin{array}{c}\text { BAS } \\
\left(10^{3} /\right. \\
\mu \mathrm{L})\end{array}$ \\
\hline & & $\mathrm{X} \pm \mathrm{SD}$ & $\mathrm{X} \pm \mathrm{SD}$ & $\mathrm{X} \pm \mathrm{SD}$ & $\mathrm{X} \pm \mathrm{SD}$ & $X \pm S D$ & $\mathrm{X} \pm \mathrm{SD}$ & $\mathrm{X} \pm \mathrm{SD}$ & $\mathrm{X} \pm \mathrm{SD}$ & $X \pm S D$ & $\mathrm{X} \pm \mathrm{SD}$ & $X \pm S D$ & $X \pm S D$ & $\begin{array}{l}X \pm \\
\text { SD }\end{array}$ \\
\hline \multirow{2}{*}{ UÖ } & KG & $\begin{array}{c}15.40 \pm \\
3.40 \mathrm{a}\end{array}$ & $\begin{array}{c}10.62 \pm \\
2.64^{\mathrm{a}}\end{array}$ & $\begin{array}{c}11.52 \pm \\
2.34^{\mathrm{a}}\end{array}$ & $\begin{array}{c}34.40 \pm \\
2.20^{\mathrm{a}}\end{array}$ & $\begin{array}{c}32.82 \pm \\
4.24^{\mathrm{d}}\end{array}$ & $\begin{array}{c}10.86 \pm \\
2.23^{\mathrm{c}}\end{array}$ & $\begin{array}{c}534.45 \\
\pm 56.50^{\text {e }}\end{array}$ & $\begin{array}{c}33.52 \pm \\
3.46^{\mathrm{a}}\end{array}$ & $\begin{array}{l}2.32 \pm \\
0.64^{\mathrm{e}}\end{array}$ & $\begin{array}{l}0.82 \pm \\
0.02^{\mathrm{e}}\end{array}$ & $\begin{array}{l}5.42 \pm \\
0.82^{\mathrm{a}}\end{array}$ & $\begin{array}{l}1.30 \pm \\
0.60^{\mathrm{a}}\end{array}$ & - \\
\hline & DG & $\begin{array}{c}15.68 \pm \\
3.30^{\mathrm{a}}\end{array}$ & $\begin{array}{c}10.49 \pm \\
2.48^{\mathrm{a}}\end{array}$ & $\begin{array}{c}11.34 \pm \\
2.23^{\mathrm{a}}\end{array}$ & $\begin{array}{c}34.00 \pm \\
2.10^{\mathrm{a}}\end{array}$ & $\begin{array}{c}32.64 \pm \\
4.23^{\mathrm{d}}\end{array}$ & $\begin{array}{c}10.82 \pm \\
2.36^{\mathrm{c}}\end{array}$ & $\begin{array}{c}540.32 \\
\pm 63.40^{\mathrm{e}}\end{array}$ & $\begin{array}{c}33.38 \pm \\
3.54^{\mathrm{a}}\end{array}$ & $\begin{array}{l}2.43 \pm \\
0.56^{\mathrm{e}}\end{array}$ & $\begin{array}{l}0.84 \pm \\
0.03^{e}\end{array}$ & $\begin{array}{l}5.36 \pm \\
0.94^{\mathrm{a}}\end{array}$ & $\begin{array}{l}1.34 \pm \\
0.58^{\mathrm{a}}\end{array}$ & \\
\hline \multirow{2}{*}{$\begin{array}{l}\text { US } 1^{\text {st }} \\
\text { Day }\end{array}$} & KG & $\begin{array}{c}11.30 \pm \\
2.10^{\mathrm{b}}\end{array}$ & $\begin{array}{l}8.44 \pm \\
1.60^{\mathrm{b}}\end{array}$ & $\begin{array}{l}9.48 \pm \\
2.12^{\mathrm{b}}\end{array}$ & $\begin{array}{c}28.32 \pm \\
2.10^{\mathrm{b}}\end{array}$ & $\begin{array}{c}33.50 \pm \\
3.50^{\mathrm{d}}\end{array}$ & $\begin{array}{l}11.28 \pm \\
2.16^{\mathrm{bc}}\end{array}$ & $\begin{array}{r}618.27 \\
\pm 48.20^{d} \\
\end{array}$ & $\begin{array}{c}33.48 \pm \\
2.46^{\mathrm{a}}\end{array}$ & $\begin{array}{l}3.23 \pm \\
0.52^{\mathrm{d}}\end{array}$ & $\begin{array}{c}0.92 \pm \\
0.04\end{array}$ & $\begin{array}{l}5.36 \pm \\
0.74^{\mathrm{a}}\end{array}$ & $\begin{array}{l}1.20 \pm \\
0.50^{\mathrm{a}}\end{array}$ & - \\
\hline & DG & $\begin{array}{c}10.20 \pm \\
2.30^{c}\end{array}$ & $\begin{array}{l}7.06 \pm \\
1.34^{\text {bc }}\end{array}$ & $\begin{array}{l}8.02 \pm \\
2.04^{\mathrm{bc}}\end{array}$ & $\begin{array}{c}25.10 \pm \\
2.30^{\mathrm{c}}\end{array}$ & $\begin{array}{c}35.58 \pm \\
3.35^{\mathrm{c}}\end{array}$ & $\begin{array}{c}11.35 \pm \\
2.08^{\mathrm{bc}}\end{array}$ & $\begin{array}{c}818.48 \pm \\
66.80^{c}\end{array}$ & $\begin{array}{c}31.76 \pm \\
2.28^{\mathrm{c}}\end{array}$ & $\begin{array}{l}3.83 \pm \\
0.68^{\text {cd }}\end{array}$ & $\begin{array}{l}1.12 \pm \\
0.08^{c}\end{array}$ & $\begin{array}{l}4.06 \pm \\
0.52^{\mathrm{b}}\end{array}$ & $\begin{array}{l}0.88 \pm \\
0.56^{\mathrm{b}}\end{array}$ & \\
\hline \multirow{2}{*}{$\begin{array}{l}\text { US } 2^{\text {nd }} \\
\text { Day }\end{array}$} & KG & $\begin{array}{l}8.30 \pm \\
1.40^{\mathrm{d}}\end{array}$ & $\begin{array}{l}8.00 \pm \\
1.44^{\mathrm{b}}\end{array}$ & $\begin{array}{l}8.56 \pm \\
1.40^{\mathrm{bc}}\end{array}$ & $\begin{array}{c}26.00 \pm \\
1.40^{\mathrm{c}}\end{array}$ & $\begin{array}{c}32.52 \pm \\
2.48^{\mathrm{d}}\end{array}$ & $\begin{array}{c}10.76 \pm \\
2.32^{\mathrm{c}}\end{array}$ & $\begin{array}{c}622.30 \\
\pm 42.40^{\mathrm{d}}\end{array}$ & $\begin{array}{c}32.94 \pm \\
2.36^{\mathrm{b}}\end{array}$ & $\begin{array}{l}4.43 \pm \\
0.46^{c}\end{array}$ & $\begin{array}{c}0.93 \pm \\
0.03^{\mathrm{d}}\end{array}$ & $\begin{array}{l}4.04 \pm \\
0.41^{\mathrm{b}}\end{array}$ & $\begin{array}{l}1.10 \pm \\
0.52^{\mathrm{a}}\end{array}$ & - \\
\hline & DG & $\begin{array}{c}7.20 \pm \\
1.30^{\mathrm{e}}\end{array}$ & $\begin{array}{c}6.42 \pm \\
1.52^{c}\end{array}$ & $\begin{array}{c}7.34 \pm \\
1.28^{c}\end{array}$ & $\begin{array}{c}23.30 \pm \\
1.52^{\mathrm{d}}\end{array}$ & $\begin{array}{c}36.32 \pm \\
2.60^{\mathrm{bc}}\end{array}$ & $\begin{array}{c}11.46 \pm \\
2.14^{\mathrm{bc}}\end{array}$ & $\begin{array}{c}934.40 \\
\pm 74.50^{\mathrm{b}}\end{array}$ & $\begin{array}{c}31.48 \pm \\
2.13^{c}\end{array}$ & $\begin{array}{l}5.48 \pm \\
0.34^{\mathrm{b}}\end{array}$ & $\begin{array}{l}1.38 \pm \\
0.07^{\mathrm{b}}\end{array}$ & $\begin{array}{c}3.16 \pm \\
0.32^{c}\end{array}$ & $\begin{array}{l}0.54 \pm \\
0.40^{c}\end{array}$ & \\
\hline \multirow{2}{*}{$\begin{array}{l}{\text { US } 3^{\text {rd }}}^{\text {Day }}\end{array}$} & KG & $\begin{array}{l}7.88 \pm \\
1.10^{\mathrm{de}} \\
\end{array}$ & $\begin{array}{l}6.54 \pm \\
1.23^{\mathrm{c}}\end{array}$ & $\begin{array}{l}8.30 \pm \\
1.20^{\mathrm{bc}}\end{array}$ & $\begin{array}{c}25.30 \pm \\
1.05^{c}\end{array}$ & $\begin{array}{c}38.67 \pm \\
2.58^{\mathrm{b}}\end{array}$ & $\begin{array}{c}12.72 \pm \\
2.18^{\mathrm{b}}\end{array}$ & $\begin{array}{r}626.40 \\
\pm 34.50^{\mathrm{d}} \\
\end{array}$ & $\begin{array}{c}32.82 \pm \\
2.18^{\mathrm{b}}\end{array}$ & $\begin{array}{l}5.40 \pm \\
0.28^{\mathrm{b}}\end{array}$ & $\begin{array}{c}0.90 \pm \\
04^{\mathrm{d}}\end{array}$ & $\begin{array}{c}3.12 \pm \\
0.26^{c}\end{array}$ & $\begin{array}{l}1.00 \pm \\
0.30^{\mathrm{ab}}\end{array}$ & - \\
\hline & DG & $\begin{array}{c}6.72 \pm \\
1.30^{f}\end{array}$ & $\begin{array}{c}4.48 \pm \\
1.34^{\mathrm{d}}\end{array}$ & $\begin{array}{c}6.30 \pm \\
1.08^{\mathrm{d}}\end{array}$ & $\begin{array}{c}20.10 \pm \\
1.00^{\mathrm{d}}\end{array}$ & $\begin{array}{c}44.82 \pm \\
3.48^{\mathrm{a}}\end{array}$ & $\begin{array}{c}14.08 \pm \\
2.04^{\mathrm{a}}\end{array}$ & $\begin{array}{c}998.40 \pm \\
98.40^{\mathrm{a}}\end{array}$ & $\begin{array}{c}31.38 \pm \\
2.10^{c}\end{array}$ & $\begin{array}{c}6.48 \pm \\
0.32^{\mathrm{a}}\end{array}$ & $\begin{array}{c}1.62 \pm \\
0.06^{a}\end{array}$ & $\begin{array}{c}1.23 \pm \\
0.38^{\mathrm{d}}\end{array}$ & $\begin{array}{c}0.30 \pm \\
0.32^{\mathrm{d}}\end{array}$ & - \\
\hline
\end{tabular}

a-f The difference between the averages of the control groups statistics $(p<0,05)$. KG: Control group, DG: Experiment group, UO: carrying different letters in the same column is important in terms of 
MCV and MCH levels were significantly higher $(\mathrm{p}<0.05)$ in terms of time in terms of statistical significance in both groups when compared to the NL average, but it was observed that this increase was higher in DG animals and statistically significant $(p<0.05)$. On the contrary, it was found that the mean levels of MCHC were decreased in both groups according to NDP, and the lowest levels were obtained in DG animals, although this decrease was statistically significant ( $p<0.05)$.

\section{Metabolic profiles}

The mean values of the blood serum biochemical parameters obtained in UO and US days in KG and DG animals are shown in Table 3. When this table is examined; The mean levels of AST, ALP, SDH, LDH, GLDH and CK enzymes decreased gradually at 3 days, gradually decreasing with time in both groups. It was statistically significant ( $\mathrm{p}<0.05$ ) in terms of all time periods in
DG animals levels were found to be low. When compared with UO, these enzymes were not statistically different between the groups ( $p>0.05$ ). From TP perspective; It was found that the highest levels were obtained from the measurements in this period but the TP concentration averages after the treatment decreased with time and the decrease in theophylline-treated DG was not statistically significant compared to the KG ( $p>$ 0.05). As the opposite situation; ALB concentration levels were lower in both groups and there was no difference in group mean ( $p>0.05$ ) in terms of group mean, but ALB concentration levels which were lower in UO gradually increased in the later time periods and reached the highest level on the third day, There was no statistically significant difference $(p>0.05)$ in terms of ALB concentration levels between day and day. Compared with UO, ALB levels were found to be higher in the later time periods and this height was statistically significant $(\mathrm{p}<0.05)$. The mean values of GLU concentrations reached to the highest level on day 3 with progressive increase in time in KG and DG animals ( $p<0.05$ ) higher than the mean of the GL animals in the US over the entire time period of the US in terms of the GLU averages.

Table 3: Metabolic profile findings.

\begin{tabular}{|c|c|c|c|c|c|c|c|c|c|c|c|c|}
\hline \multirow[t]{2}{*}{ Time } & \multirow{2}{*}{ Groups } & $\begin{array}{c}\text { AST } \\
\text { (IU/L) }\end{array}$ & $\begin{array}{c}\text { SDH } \\
\text { (IU/L) }\end{array}$ & $\begin{array}{c}\text { ALP } \\
\text { (IU/L) }\end{array}$ & $\begin{array}{c}\text { LDH } \\
\text { (IU/L) }\end{array}$ & $\begin{array}{l}\text { GLDH } \\
\text { (IU/L) }\end{array}$ & $\begin{array}{c}\text { CK (mg/ } \\
\text { dL) }\end{array}$ & $\begin{array}{c}\text { TP (g/ } \\
\text { dL) }\end{array}$ & $\begin{array}{c}\text { ALB (g/ } \\
\text { dL) }\end{array}$ & $\begin{array}{c}\text { GLU } \\
\text { (mg/dL) }\end{array}$ & $\begin{array}{c}\text { TB (mg/ } \\
\text { dL) }\end{array}$ & $\begin{array}{c}\text { DB(mg/ } \\
\text { dL) }\end{array}$ \\
\hline & & $\mathrm{X} \pm \mathrm{SD}$ & $\mathrm{X} \pm \mathrm{SD}$ & $\mathrm{X} \pm \mathrm{SD}$ & $\mathrm{X} \pm \mathrm{SD}$ & $\mathrm{X} \pm \mathrm{SD}$ & $\mathrm{X} \pm \mathrm{SD}$ & $\mathrm{X} \pm \mathrm{SD}$ & $\mathrm{X} \pm \mathrm{SD}$ & $\mathrm{X} \pm \mathrm{SD}$ & $\mathrm{X} \pm \mathrm{SD}$ & $\mathrm{X} \pm \mathrm{SD}$ \\
\hline \multirow{2}{*}{ UÖ } & KG & $\begin{array}{c}188.28 \pm \\
34.12^{\mathrm{a}}\end{array}$ & $\begin{array}{c}13.27 \pm \\
2.44^{\mathrm{b}}\end{array}$ & $\begin{array}{c}214.40 \pm \\
45.66^{\mathrm{a}}\end{array}$ & $\begin{array}{c}1740.42 \\
\pm \\
288.30^{a}\end{array}$ & $\begin{array}{c}478.32 \pm \\
86.24^{\mathrm{a}}\end{array}$ & $\begin{array}{l}76.48 \pm \\
8.421^{\mathrm{a}}\end{array}$ & $\begin{array}{c}10.42 \pm \\
0.40^{\mathrm{a}}\end{array}$ & $\begin{array}{l}2.77 \pm \\
0.64 \mathrm{~b}\end{array}$ & $\begin{array}{l}4.86 \pm \\
0.65 \mathrm{e}\end{array}$ & $\begin{array}{l}0.56 \pm \\
0.04^{\mathrm{a}}\end{array}$ & $\begin{array}{l}0.17 \pm \\
0.00^{\mathrm{a}}\end{array}$ \\
\hline & DG & $\begin{array}{c}186.37 \pm \\
35.14^{\mathrm{a}}\end{array}$ & $\begin{array}{c}13.08 \pm \\
2.36^{\mathrm{b}}\end{array}$ & $\begin{array}{c}216.00 \pm \\
50.10^{\mathrm{a}}\end{array}$ & $\begin{array}{c}1734.12 \\
\pm \\
282.40^{a}\end{array}$ & $\begin{array}{c}463.47 \pm \\
90.31^{\mathrm{a}}\end{array}$ & $\begin{array}{l}75.23 \pm \\
9.42^{\mathrm{a}}\end{array}$ & $\begin{array}{c}10.38 \pm \\
0.36^{\mathrm{a}}\end{array}$ & $\begin{array}{l}2.75 \pm \\
0.56^{\mathrm{b}}\end{array}$ & $\begin{array}{l}4.92 \pm \\
0.73^{\mathrm{e}}\end{array}$ & $\begin{array}{l}0.55 \pm \\
0.05^{\mathrm{a}}\end{array}$ & $\begin{array}{c}0.18 \pm \\
0.001^{\mathrm{a}}\end{array}$ \\
\hline \multirow{2}{*}{$\begin{array}{l}\text { US } 1^{\text {st }} \\
\text { Day }\end{array}$} & $\mathrm{KG}$ & $\begin{array}{c}144.26 \pm \\
28.36^{\mathrm{c}}\end{array}$ & $\begin{array}{c}10.34 \pm \\
1.48^{\text {cd }}\end{array}$ & $\begin{array}{c}186.28 \pm \\
32.44^{\mathrm{b}}\end{array}$ & $\begin{array}{c}1320.18 \\
\pm \\
114.20^{\mathrm{e}} \\
\end{array}$ & $\begin{array}{c}341.16 \pm \\
47.20^{\mathrm{d}}\end{array}$ & $\begin{array}{c}54.27 \pm \\
6.44^{c}\end{array}$ & $\begin{array}{l}9.47 \pm \\
0.32^{\mathrm{b}}\end{array}$ & $\begin{array}{l}2.95 \pm \\
0.56^{\mathrm{ab}}\end{array}$ & $\begin{array}{l}5.13 \pm \\
0.46^{d}\end{array}$ & $\begin{array}{l}0.49 \pm \\
0.03^{\mathrm{ab}}\end{array}$ & $\begin{array}{l}0.11 \pm \\
0.00^{\mathrm{b}}\end{array}$ \\
\hline & DG & $\begin{array}{c}164.48 \pm \\
32.12^{\mathrm{b}}\end{array}$ & $\begin{array}{c}12.22 \pm \\
2.68^{\mathrm{bc}}\end{array}$ & $\begin{array}{c}214.30 \pm \\
68.15^{\mathrm{a}}\end{array}$ & $\begin{array}{c}1610.37 \\
\pm \\
146.12^{\mathrm{b}}\end{array}$ & $\begin{array}{c}421.18 \pm \\
94.13^{\mathrm{b}}\end{array}$ & $\begin{array}{c}75.16 \pm \\
7.24^{\mathrm{a}}\end{array}$ & $\begin{array}{l}8.04 \pm \\
0.38^{c}\end{array}$ & $\begin{array}{l}2.91 \pm \\
0.44^{\mathrm{ab}}\end{array}$ & $\begin{array}{c}6.16 \pm \\
0.60^{c}\end{array}$ & $\begin{array}{l}0.48 \pm \\
0.04^{\mathrm{ab}}\end{array}$ & $\begin{array}{l}0.10 \pm \\
0.00^{\mathrm{b}}\end{array}$ \\
\hline \multirow{2}{*}{$\begin{array}{l}{\text { US } 2^{\text {nd }}}^{\text {Day }}\end{array}$} & $\mathrm{KG}$ & $\begin{array}{c}118.36 \pm \\
16.10^{\mathrm{d}} \\
\end{array}$ & $\begin{array}{l}9.23 \pm \\
1.68^{\mathrm{d}} \\
\end{array}$ & $\begin{array}{c}148.22 \pm \\
32.14^{\mathrm{c}}\end{array}$ & $\begin{array}{r}830.22 \pm \\
108.30^{\mathrm{f}}\end{array}$ & $\begin{array}{c}264.14 \pm \\
44.12^{\mathrm{e}} \\
\end{array}$ & $\begin{array}{r}44.23 \pm \\
6.48^{\mathrm{c}} \\
\end{array}$ & $\begin{array}{r}8.32 \pm \\
0.30^{c}\end{array}$ & $\begin{array}{c}3.05 \pm \\
0.32 \mathrm{ab}\end{array}$ & $\begin{array}{c}6.18 \pm \\
0.37^{c}\end{array}$ & $\begin{array}{l}0.39 \pm \\
0.03^{b}\end{array}$ & $\begin{array}{l}0.08 \pm \\
0.00^{c}\end{array}$ \\
\hline & DG & $\begin{array}{c}144.13 \pm \\
27.26^{c}\end{array}$ & $\begin{array}{c}13.44 \pm \\
3.04^{\mathrm{b}}\end{array}$ & $\begin{array}{c}212.28 \pm \\
63.44^{\mathrm{a}}\end{array}$ & $\begin{array}{c}1442.36 \\
\pm \\
134.26^{\mathrm{c}}\end{array}$ & $\begin{array}{c}394.17 \pm \\
77.48^{\mathrm{c}}\end{array}$ & $\begin{array}{c}72.12 \pm \\
6.14^{\mathrm{a}}\end{array}$ & $\begin{array}{l}7.03 \pm \\
0.28^{\mathrm{d}}\end{array}$ & $\begin{array}{l}3.03 \pm \\
0.40^{\mathrm{ab}}\end{array}$ & $\begin{array}{l}7.14 \pm \\
0.34^{\mathrm{b}}\end{array}$ & $\begin{array}{l}0.37 \pm \\
0.03^{b}\end{array}$ & $\begin{array}{l}0.09 \pm \\
0.00^{c}\end{array}$ \\
\hline \multirow{2}{*}{$\begin{array}{l}\text { US }^{\text {rd }} \\
\text { Day }\end{array}$} & $\mathrm{KG}$ & $\begin{array}{l}96.32 \pm \\
13.27^{\mathrm{e}}\end{array}$ & $\begin{array}{l}8.36 \pm \\
0.78^{\mathrm{d}}\end{array}$ & $\begin{array}{c}112.24 \pm \\
13.60^{\mathrm{d}}\end{array}$ & $\begin{array}{c}650.24 \pm \\
72.46^{\mathrm{g}}\end{array}$ & $\begin{array}{c}232.26 \pm \\
33.74^{\mathrm{f}}\end{array}$ & $\begin{array}{c}34.13 \pm \\
7.12^{\mathrm{e}}\end{array}$ & $\begin{array}{r}7.90 \pm \\
0.30^{c}\end{array}$ & $\begin{array}{l}3.34 \pm \\
0.24^{\mathrm{a}}\end{array}$ & $\begin{array}{c}7.32 \pm \\
0.27^{b}\end{array}$ & $\begin{array}{l}0.23 \pm \\
0.02^{\mathrm{c}}\end{array}$ & $\begin{array}{l}0.06 \pm \\
0.00^{\mathrm{d}}\end{array}$ \\
\hline & DG & $\begin{array}{c}123.44 \pm \\
23.48^{\mathrm{d}}\end{array}$ & $\begin{array}{c}16.76 \pm \\
2.38^{\mathrm{a}}\end{array}$ & $\begin{array}{c}215.30 \pm \\
58.70^{\mathrm{a}}\end{array}$ & $\begin{array}{c}1284.23 \\
\pm \\
114.22^{\mathrm{d}}\end{array}$ & $\begin{array}{c}338.34 \pm \\
64.18^{\mathrm{d}}\end{array}$ & $\begin{array}{c}64.13 \pm \\
6.22^{\mathrm{b}}\end{array}$ & $\begin{array}{l}6.83 \pm \\
0.24^{\mathrm{e}}\end{array}$ & $\begin{array}{l}3.26 \pm \\
0.28^{\mathrm{a}}\end{array}$ & $\begin{array}{c}8.24 \pm \\
0.32^{\mathrm{a}}\end{array}$ & $\begin{array}{l}0.24 \pm \\
0.02^{c}\end{array}$ & $\begin{array}{l}0.06 \pm \\
0.00^{\mathrm{d}}\end{array}$ \\
\hline
\end{tabular}

a-g The difference between the averages of the control groups carrying different letters in the same column is important in terms of statistics $(\mathrm{p}<0,05)$. KG: Control group, DG: Experiment group, UO: Before experiment, US: After experiment.

In terms of $\mathrm{TB}$ and $\mathrm{DB}$ concentrations, there was no statistically significant difference between the groups or time periods ( $p>0.05$ ), while the high TB and DB concentration levels decreased with time and reached to the lowest level in US 3 days and the UO and US concentration levels $(\mathrm{p}<0.05)$.

\section{Discussion}

Theophylline remains one of the widely prescribed drugs worldwide for the treatment of chronic obstructive pulmonary disease (COPD) due to its low cost and easy access, as well as its antiinflammatory effect, which relaxes the airway smooth muscles [20]. This includes the reduction of chemoreceptor response to hypoxia and hypercapnia, the active reflexes acting by stimulation of the posterior pharynx, and the role of ineffective ventilation due to the impaired coordination of the inspiratory muscles and upper respiratory muscles [21,22].

In our study, the effects of theophylline on the respiratory system were investigated in a 3-day trial, and in studies of 


\section{Juniper Online Journal of Case Studies}

the adequacy of the study period, some investigators noted that theophylline increased rapidly in clinical symptoms and increased ventilation in the presence of apnea and that theophylline did not differ from $[23,24]$.

While theophylline is used as an adjunctive therapeutic agent to treat bovine respiratory disease, there may be risks associated with typhoid [25,26]. Some authors [16,27-29] reported that symptoms such as tachycardia, tachycardia, vomiting, diarrhea, reflux and muscle spasms could be formed. In our study, significant increases in respiratory and cardiac frequencies in DG animals following the application of theophylline (Table 1) were consistent with those reported by these investigators. In our study, in addition to the above-mentioned klinink symptoms, 5 animals in the group of theophylline-treated DG, which had no KG animals, apparently observed vibrations in the costoabdominal region and in the foot muscles. As a matter of fact, it has been reported that contractions in the intercostal and strenal muscles are formed in the ice of $100-400 \mathrm{mg}$ dose by oral route, and that the possible cause is related to the hypofolicity of theophylline [27,30,31].

As a result of extensive literature reviews, we could not find a direct study of the effect of theophylline on hematological parameters on the ice. However, theophylline has been reported to weaken erythropoietin production and reduce RBC levels in some normal subjects and in patients developing erythrocytosis $[32,33]$. Bakris et al. [32] reported that theophylline also caused a decrease in hematocrit levels [33], similarly reporting a decrease in blood erythrocyte levels following theophylline administration. Ohnuma et al. [34] reported that the use of theophylline resulted in an increase in the platelet count. Considering the averages of the haematological examination parameters measured in our study, the RBC, HB, and HTC averages were significantly lower after the theophylline administration in DG animals compared to $\mathrm{KG}$, consistent with the reported findings of the investigators.

Ubbink et al. [33] reported that after the administration of theophylline, a significant reduction in the levels of enzymes such as AST and ALT was found. Findings similar to this study were also obtained in a study in rats given $20 \mathrm{mg} / \mathrm{kg} /$ day theophylline [35]. Khidr et al. [37] found that catalase enzymes increased on day 4 , while the study of snails showed a similar decrease in AST, ALT and LDH levels in the group of theophylline. For 3 days during the work we did i.v. The decrease in AST, ALP, LDH, GLDH, SDH and CK enzyme levels measured in the same manner in the animals treated with theophylline is parallel to that reported by the researchers mentioned.

In our study, the probable cause of high baseline TB and DB levels was due to maternal erythrocyte destruction, the decreasing levels were not related to theophylline, but the difference between TB and DB concentrations in BSE and BSE animals. Kato et al. [28] report that low and short-term use of theophylline may lead to an increase in blood enzyme levels of theophylline, which does not lead to a significant increase but reaches high plasma concentrations and / or is used for a long time.
In our study, we observed a decrease in TP levels following theophylline administration in DG animals. In a study of mice showing asthma-like respiratory problems [38], it was found that there was a significant decrease in the rate of theophylline administration while having a high TP rate in lavage fluids. Edmunds \& Mayhew [16] also reported that theophylline use at high doses may lead to proteinuria. Bruguerolle \& Dubus [39] claimed that high fever caused an increase in blood protein and albumin levels and that theophylline administration did not cause a change in TP and ALB levels in high-fever subjects [37], in their study of snails, causing them to decrease slightly.

In the study we did, KG and DG were observed to have increased levels of GLU in animals, which was higher in DG animals. This finding is consistent with the findings of researchers [40] who report that theophylline in hypoglycemic subjects leads to an increase in blood GLU levels by increasing the level of cAMP. Raguso et al. [29] reported that theophylline infusions lead to significant increases in blood GLU levels in normal subjects.

As a result; it has been determined that theophylline investigated for the first time in the calves can provide significant improvements in the clinical symptoms of the respiratory system and contribute to recovery.

\section{References}

1. Wiseman A, Msolla PM, Selman IE, Allan EM, Pirie HM (1980) Clinical and epidemiological features of 15 incidents of severe infectious bovine rhinotracheitis. Vet Rec 107(19): 436-441.

2. Andwers AH (2004) Calf enteritis diarrhoea in the pre-weaned calfstrategic investigation of outbreaks. Cattle Practice 12(2): 109-114.

3. Barker IK, Van Dreumel AA, Palmer N (1993) The alimentary system. In: Jubb KVF, Kennedy PC, Palmer N (Eds.), Pathology of domestic animals. $\left(4_{\text {th }}\right.$ edn), Vol 2, Acdemic Press, San Diego, USA, pp. 1-300.

4. Bartels CJ, Holzhauer M, Jorritsma R, Swart WA, Lam TJ (2010) Prevalence, prediction and risk factors of enteropathogens in normal and non-normal faeces of young Dutch dairy calves. Prev Vet Med 93(2-3): 162-169.

5. Blood DC, Radostsit OM (1989) Veterinary Medicine. ( $7^{\text {th }}$ edn), Bailliere Tindall, London, UK.

6. Dorchies J, Duncan B, Losson JP, Alzıe U (2012) Parasitologie Clinique des bovins. Med Com Editions, France, pp. 341-362.

7. Cho YI, Yoon KJ (2014) An overview of calf diarrhea-infectious etiology, diagnosis, and intervention. J Vet Sci 15(1): 1-17.

8. Emre Z, Fidancı H (1998) Prevalence of mix infections of Cryptosporidium spp., Escherichia coli K99 and Rotavirus in the faeces of diarrhoeic and healthy cattle in Ankara, Turkey and in vitro resistance of Escherichia coli K99 to antimicrobial agents. Turkish Journal of Veterinary and Animal Science 22: 175-178.

9. Hur TY, Jung YH, Choe CY, Cho YI, Kang SJ, et al. (2013) The dairy calf mortality: the causes of calf death during ten years at a large dairy farm in Korea, Korean J Vet Res 53: 103-108.

10. Cho YI, Han JI, Wang C, Cooper V, Schwartz K, et al. (2013) Case-control study of microbiological etiology associated with calf diarrhea. Vet Microbiol 166(3-4): 375-385.

11. Mcfadden ER (1991) Methylxanthines in the treatment of asthma: the rise, the fall, and the possible rise again. Ann Intern Med 115(4): 323 324. 
12. Persson CG, Draco AB (1988) Xanthines as airway anti inflammatory drugs. J Allergy Clin Immunol 81: 615-617

13. Sullivan P, Bekir S, Jaffar Z, Page C, Jeffery P, et al. (1994) Antiinflammatory effects of low-dose oral theophylline in atopic asthma. Lancet 343(8904): 1006-1008.

14. Elenbaas RM, Payne VW (1984) Prediction of serum theophylline levels. Ann Emerg Med 13(2): 92-96.

15. ÇevikY, Kavalcı C, Daş M, İzdeşS (2010) Severe theophyllineintoxication, rhabdomyolysis, disseminated intravascular coagulopathy and death: Case report. Akatos 1: 24-27

16. Edmunds MW, Mayhew MS (2009) Pharmacology for the Primary Care Provider. ( $3^{\text {rd }}$ edn), Mosby Elsevier, USA.

17. Giguere S, Slade JK, Sanchez LC (2008) Retrospective comparison of caffeine and doxapram for the treatment of hypercapnia in foals with hypoxic-ischemic encephalopathy. J Vet Int Med 22(2): 401-405.

18. Charehsaz M, Gürbay A, Karakılıç E, Şahin G (2011) Teofilin ile zehirlenmeler ve tedavi yaklaşımı. J Clin Anal Med 2(3): 157-163

19. Erden IA, Pamuk AG, Turgut HC, Altınel S, Akıncı SB, et al. (2010) Olgu sunumu ve literatürün gözden geçirilmesi: Yaşlılarda zehirlenme. Akad Geriatri Derg 2(1): 54-57

20. Barnes PJ (2009) Theophylline. Asthma and COPD, (2nd edn), Academic Press, USA, pp. 627-638

21. Darnall RA (2010) The role of CO2 and central chemoreception in the control of breathing in the fetus and the neonate. Respir Physiol Neurobiol 173(3): 201-212.

22. Praud JP (2010) Upper airway reflexes in response to gastric reflux. Paediatr Respir Rev 11: 208-12.

23. Henderson-Smart DJ, Steer PA (2010) Caffeine versus theophylline for apnea in preterm infants. Cochrane Database Syst Rev (1): CD000273.

24. Bleul U, Bircher B, Jud RS, Kutter AP (2010) Respiratory and cardiovascular effects of doxapram and theophylline for the treatment of asphyxia in neonatal calves. Theriogenology 73(5): 612-619.

25. Langston VC, Koritz GD, Davis LE, Neff-Davis C (1989) Pharmacokinetic properties of theophylline given intravenously and orally to ruminating calves. Am J Vet Res 50(4): 493-497.

26. Arnold JD, Courtenay-Evans RJ, Whitfield R, O’Reilly JF, Petrie GR, et al. (1990) Comparative assessment of enprofylline and theophylline for chronic obstructive airways disease in the elderly. Respir Med 84(3): 211-215

27. Ray A, Gulati K, Anand S, Vijayan VK (2005) Pharmacological studies on the mechanisms of aminophylline-induced seizures in rats. Indian J Exp Biol 43(10): 849-853.
28. Kato Z, Yamagishi A, Nakamura M, Kondo N (2009) Theophyllineassociated status epilepticus in an infant: Pharmacokinetics and the risk of suppository use. World Journal of Pediatrics 5(4): 316-318.

29. Raguso CA, Coggan AR, Sidossis LS, Gastaldelli A, Wolfe RR (1996) Effect of Theophylline on Substrate Metabolism During Exercise. Metabolism 45(9): 1153-1160.

30.Gulati K, Ray A, Pal G, Vijayan VK (2005) Possible role of free radicals in theophylline induced seizures in mice. Pharmacol Biochem Behav 82(1): 241-245.

31. Gulatı K, Ray A, Pal R, Vıjayan VK (2007) Free Radicals in drug toxicity: focus on theophylline. In: Ray A, Gulati K, editiors. Current Trends in Pharmacology. New Delhi: IK International, India, pp 443-459.

32. Bakris GL, Sauter ER, Hussey JL, Fisher JW, Gaber AO, et al. (1990) Effects of theophylline on erythropoietin production in normal subjects and in patients with erythrocytosis after renal transplantation. $\mathrm{N}$ Engl J Med 323(2): 86-90.

33. Philip-Joet F, Bruguerolle B, Reynaud M, Arnaud A (1992) Correlations between theophylline concentrations in plasma, erythrocytes and cantharides-induced blister fluid and peak expiratory flow in asthma patients. Eur J Clin Pharmacol 43(5): 563-571.

34. Ubbink JB, Vermaak WJ, Delport R, Serfontein WJ, Bartel P (1990) The relationship between vitamin B6 metabolism, asthma, and theophylline therapy. Ann N Y Acad Sci 585: 285-294.

35. Ohnuma O, Shirata Y, Miyazawa K (1988) Use of theophylline in the investigation of pseudothrombocytopenia induced by edetic acid (EDTA-2K). J Clin Pathol 41(8): 915-917.

36. Hussein RM, Elsirafy OM, Wahba YS, Kawy HS, Hasanin AH, et al. (2015) Theophylline, an old drug with multi-faceted effects: Its potential benefits in immunological liver injury in rats. Life Sci 136: 100-107.

37.Khıdr FK, Abo Hashem AAM, Keshta TMS, Ismaıl SMA (2011) Some of biochemical changes induced by Theophylline and Furosemide in the land snail, Monacha obstructa. J Plant Prot and Pathology Mansoura Univ 2(4): 429-437.

38.Yu B, He Q, Gao Z (1998) The role of glucocorticosteroid and theophylline in asthmatic inflammation of murine model and the inhibition in NO production in lung. Zhonghua Jie He He Hu Xi Za Zhi 21(11): 664-669.

39. Bruguerolle B, Dubus JC (1993) Fever-induced changes in theophylline pharmacokinetics in rats. Fundam Clin Pharmacol 7(8): 429-433.

40.Hvidberg A, Rasmussen MH, Christensen NJ, Hilsted J (1994) Theophylline enhances glucose recovery after hypoglycemia in healthy 
This work is licensed under Creative Commons Attribution 4.0 License DOI: 10.19080/JOJCS.2018.08.555736

\section{Your next submission with Juniper Publishers} will reach you the below assets

- Quality Editorial service

- Swift Peer Review

- Reprints availability

- E-prints Service

- Manuscript Podcast for convenient understanding

- Global attainment for your research

- Manuscript accessibility in different formats

( Pdf, E-pub, Full Text, Audio)

- Unceasing customer service

Track the below URL for one-step submission

https://juniperpublishers.com/online-submission.php 